Sean K. Gorman

Michelle Ho Chung

Richard S. Slavik

Peter J. Zed

Kerry Wilbur

Vinay K. Dhingra

\section{Erratum to: A critical appraisal of the quality of critical care pharmacotherapy clinical practice guidelines and their strength of recommendations}

Published online: 24 August 2010

(C) Copyright jointly held by Springer and ESICM 2010

The online version of the original article can be found under doi:10.1007/s00134-010-1786-8.

Electronic supplementary material The online version of this article (doi:10.1007/s00134-010-1976-4) contains supplementary material, which is available to authorized users.

\section{S. K. Gorman (®) · P. J. Zed}

Department of Pharmacy, Capital District Health Authority, College of Pharmacy, Dalhousie University,

c/o Rm 2043 Victoria Building, 1276 South Park Street,

Halifax, NS B3H 2Y9, Canada

e-mail: sean.gorman@dal.ca

Tel.: +1-902-4736576

Fax: +1-902-4731606

\section{H. Chung}

Department of Pharmacy, St. Paul's Hospital, 1081 Burrard Street, Vancouver, BC V6Z 1Y6, Canada

\section{R. S. Slavik}

Pharmacy Department, Interior Health Authority,

Faculty of Pharmaceutical Sciences,

University of British Columbia, 200-1835 Gordon Drive,

Kelowna, BC V1Y 3H5, Canada

\section{P. J. Zed}

Department of Emergency Medicine, Dalhousie University, Halifax Infirmary, Suite 355 (Room 345), 1796 Summer Street, Halifax, NS B3H 3A7, Canada

\section{K. Wilbur}

College of Pharmacy, Qatar University,

P.O. Box 2713, Doha, Qatar

\section{K. Dhingra}

Intensive Care Unit, Vancouver General Hospital,

855 West 12th Avenue, Vancouver, BC V5Z 1M9, Canada

V. K. Dhingra

Faculty of Medicine, University of British Columbia,

Vancouver, BC V5Z 1M9, Canada

\section{Erratum to: Intensive Care Med DOl:10.1007/s00134-010-1786-8}

Some data from Appendix 1 were lost during the editorial process. The corrected appendix is reproduced here. Additional electronic supplementary material has also been supplied. 
Appendix 1 Clinical practice guidelines with AGREE recommendation, standardized domain scores, and breakdown of item scores

\begin{tabular}{|c|c|c|c|c|}
\hline Guideline title & $\begin{array}{l}\text { AGREE } \\
\text { recommendation }\end{array}$ & $\begin{array}{l}\text { Standardized } \\
\text { domain scores }^{\mathrm{a}}\end{array}$ & $\begin{array}{l}\text { Number of items } \\
\text { scoring } 1-2\end{array}$ & $\begin{array}{l}\text { Number of items } \\
\text { scoring 3-4 }\end{array}$ \\
\hline $\begin{array}{l}\text { Guidelines for the } \\
\text { management of penetrating } \\
\text { brain injury [9] }\end{array}$ & Not recommended & $\begin{array}{l}\text { Domain 1: } 67 \% \\
\text { Domain 2: } 25 \% \\
\text { Domain 3: } 61 \% \\
\text { Domain 4: } 58 \% \\
\text { Domain 5: } 3 \% \\
\text { Domain 6: } 8 \%\end{array}$ & 12 & 11 \\
\hline $\begin{array}{l}\text { Guidelines for the } \\
\text { management of severe } \\
\text { traumatic brain injury [10] }\end{array}$ & $\begin{array}{l}\text { Strongly } \\
\quad \text { recommended }\end{array}$ & $\begin{array}{l}\text { Domain 1: } 72 \% \\
\text { Domain 2: } 31 \% \\
\text { Domain 3: } 93 \% \\
\text { Domain 4: } 92 \% \\
\text { Domain 5: } 17 \% \\
\text { Domain 6: } 25 \%\end{array}$ & 7 & 16 \\
\hline $\begin{array}{l}\text { Blunt cerebrovascular injury } \\
\text { practice management } \\
\text { guidelines [11] }\end{array}$ & Not recommended & $\begin{array}{l}\text { Domain 1: } 67 \% \\
\text { Domain 2: } 17 \% \\
\text { Domain 3: } 57 \% \\
\text { Domain 4: } 60 \% \\
\text { Domain 5: } 8 \% \\
\text { Domain 6: } 0 \%\end{array}$ & 12 & 11 \\
\hline $\begin{array}{l}\text { Guidelines for the } \\
\text { management of } \\
\text { aneurysmal subarachnoid } \\
\text { hemorrhage [12] }\end{array}$ & Not recommended & $\begin{array}{l}\text { Domain 1: } 31 \% \\
\text { Domain 2: } 6 \% \\
\text { Domain 3: } 25 \% \\
\text { Domain 4: } 48 \% \\
\text { Domain 5: } 6 \% \\
\text { Domain 6: } 0 \%\end{array}$ & 17 & 6 \\
\hline $\begin{array}{l}\text { Guidelines for the } \\
\text { management of } \\
\text { spontaneous intracerebral } \\
\text { hemorrhage [13] }\end{array}$ & $\begin{array}{l}\text { Recommended } \\
\text { with alterations }\end{array}$ & $\begin{array}{l}\text { Domain 1: } 33 \% \\
\text { Domain 2: } 29 \% \\
\text { Domain 3: } 58 \% \\
\text { Domain 4: } 73 \% \\
\text { Domain 5: } 3 \% \\
\text { Domain 6: } 58 \%\end{array}$ & 11 & 12 \\
\hline $\begin{array}{l}\text { Guideline on the } \\
\text { management of status } \\
\text { epilepticus [14] }\end{array}$ & $\begin{array}{l}\text { Recommended } \\
\text { with alterations }\end{array}$ & $\begin{array}{l}\text { Domain 1: } 44 \% \\
\text { Domain 2: } 21 \% \\
\text { Domain 3: } 63 \% \\
\text { Domain 4: } 67 \% \\
\text { Domain 5: } 0 \% \\
\text { Domain 6: } 38 \%\end{array}$ & 11 & 12 \\
\hline $\begin{array}{l}\text { Clinical practice guidelines } \\
\text { for sustained use of } \\
\text { sedatives and analgesics in } \\
\text { the critically ill adult [15] }\end{array}$ & $\begin{array}{l}\text { Recommended } \\
\text { with alterations }\end{array}$ & $\begin{array}{l}\text { Domain 1: } 47 \% \\
\text { Domain 2: } 38 \% \\
\text { Domain 3: } 57 \% \\
\text { Domain 4: } 88 \% \\
\text { Domain 5: } 28 \% \\
\text { Domain 6: } 46 \%\end{array}$ & 9 & 14 \\
\hline $\begin{array}{l}\text { Clinical practice guidelines } \\
\text { for sustained } \\
\text { neuromuscular blockade in } \\
\text { the adult critically ill } \\
\text { patient [16] }\end{array}$ & $\begin{array}{l}\text { Recommended } \\
\text { with alterations }\end{array}$ & $\begin{array}{l}\text { Domain 1: } 50 \% \\
\text { Domain 2: } 38 \% \\
\text { Domain 3: } 61 \% \\
\text { Domain 4: } 81 \% \\
\text { Domain 5: } 25 \% \\
\text { Domain 6: } 38 \%\end{array}$ & 8 & 15 \\
\hline $\begin{array}{l}\text { Evidence-based clinical } \\
\text { practice guideline for the } \\
\text { prevention of ventilator- } \\
\text { associated pneumonia [17] }\end{array}$ & $\begin{array}{l}\text { Strongly } \\
\quad \text { recommended }\end{array}$ & $\begin{array}{l}\text { Domain 1: } 75 \% \\
\text { Domain 2: } 65 \% \\
\text { Domain 3: } 95 \% \\
\text { Domain 4: } 79 \% \\
\text { Domain 5: } 39 \% \\
\text { Domain 6: } 96 \%\end{array}$ & 4 & 19 \\
\hline $\begin{array}{l}\text { Guidelines for the } \\
\text { management of adults with } \\
\text { hospital-acquired, } \\
\text { ventilator-associated, and } \\
\text { healthcare-associated } \\
\text { pneumonia [18] }\end{array}$ & $\begin{array}{l}\text { Recommended } \\
\text { with alterations }\end{array}$ & $\begin{array}{l}\text { Domain 1: } 64 \% \\
\text { Domain 2: } 25 \% \\
\text { Domain 3: } 39 \% \\
\text { Domain 4: } 75 \% \\
\text { Domain 5: } 22 \% \\
\text { Domain 6: } 67 \%\end{array}$ & 12 & 11 \\
\hline
\end{tabular}


Appendix 1 continued

\begin{tabular}{|c|c|c|c|c|}
\hline Guideline title & $\begin{array}{l}\text { AGREE } \\
\text { recommendation }\end{array}$ & $\begin{array}{l}\text { Standardized } \\
\text { domain scores }\end{array}$ & $\begin{array}{l}\text { Number of items } \\
\text { scoring } 1-2\end{array}$ & $\begin{array}{l}\text { Number of items } \\
\text { scoring 3-4 }\end{array}$ \\
\hline $\begin{array}{l}\text { Consensus guidelines on the } \\
\text { management of } \\
\text { community-acquired } \\
\text { pneumonia in adults [19] }\end{array}$ & $\begin{array}{l}\text { Strongly } \\
\quad \text { recommended }\end{array}$ & $\begin{array}{l}\text { Domain 1: } 81 \% \\
\text { Domain 2: } 44 \% \\
\text { Domain 3: } 55 \% \\
\text { Domain 4: } 90 \% \\
\text { Domain 5: } 56 \% \\
\text { Domain 6: } 71 \%\end{array}$ & 7 & 16 \\
\hline $\begin{array}{l}\text { The American-European } \\
\text { consensus conference on } \\
\text { ARDS, part } 2[20]\end{array}$ & Not recommended & $\begin{array}{l}\text { Domain 1: } 42 \% \\
\text { Domain 2: } 19 \% \\
\text { Domain 3: } 17 \% \\
\text { Domain 4: } 35 \% \\
\text { Domain 5: } 11 \% \\
\text { Domain 6: } 0 \%\end{array}$ & 17 & 6 \\
\hline $\begin{array}{l}\text { Hospital management of } \\
\text { adults with severe acute } \\
\text { respiratory syndrome } \\
\text { (SARS) if SARS } \\
\text { re-emerges [21] }\end{array}$ & Not recommended & $\begin{array}{l}\text { Domain 1: } 44 \% \\
\text { Domain 2: } 17 \% \\
\text { Domain 3: } 13 \% \\
\text { Domain 4: } 40 \% \\
\text { Domain 5: } 0 \% \\
\text { Domain 6: } 0 \%\end{array}$ & 20 & 3 \\
\hline $\begin{array}{l}\text { Inhaled nitric oxide therapy } \\
\text { in adults: European expert } \\
\text { recommendations [22] }\end{array}$ & Not recommended & $\begin{array}{l}\text { Domain 1: } 39 \% \\
\text { Domain 2: } 19 \% \\
\text { Domain 3: } 23 \% \\
\text { Domain 4: } 40 \% \\
\text { Domain 5: } 3 \% \\
\text { Domain 6: } 83 \%\end{array}$ & 16 & 7 \\
\hline $\begin{array}{l}\text { Surviving sepsis campaign: } \\
\text { international guidelines } \\
\text { for management of severe } \\
\text { sepsis and septic } \\
\text { shock [23] }\end{array}$ & $\begin{array}{l}\text { Strongly } \\
\quad \text { recommended }\end{array}$ & $\begin{array}{l}\text { Domain 1: } 69 \% \\
\text { Domain 2: } 42 \% \\
\text { Domain 3: } 71 \% \\
\text { Domain 4: } 92 \% \\
\text { Domain 5: } 36 \% \\
\text { Domain 6: } 100 \%\end{array}$ & 6 & 17 \\
\hline $\begin{array}{l}\text { Practice parameters for } \\
\text { hemodynamic support of } \\
\text { sepsis in adult patients [24] }\end{array}$ & Not recommended & $\begin{array}{l}\text { Domain 1: } 61 \% \\
\text { Domain 2: } 38 \% \\
\text { Domain 3: } 35 \% \\
\text { Domain 4: } 71 \% \\
\text { Domain 5: } 6 \% \\
\text { Domain 6: } 4 \%\end{array}$ & 14 & 9 \\
\hline $\begin{array}{l}\text { Evidence-based colloid use } \\
\text { in the critically ill [25] }\end{array}$ & $\begin{array}{l}\text { Recommended } \\
\text { with alterations }\end{array}$ & $\begin{array}{l}\text { Domain 1: } 53 \% \\
\text { Domain 2: } 21 \% \\
\text { Domain 3: } 51 \% \\
\text { Domain 4: } 38 \% \\
\text { Domain 5: } 8 \% \\
\text { Domain 6: } 46 \%\end{array}$ & 13 & 10 \\
\hline $\begin{array}{l}\text { Guidelines for } \\
\text { cardiopulmonary } \\
\text { resuscitation and } \\
\text { emergency cardiovascular } \\
\text { care [26] }\end{array}$ & $\begin{array}{l}\text { Strongly } \\
\quad \text { recommended }\end{array}$ & $\begin{array}{l}\text { Domain 1: } 64 \% \\
\text { Domain 2: } 38 \% \\
\text { Domain 3: } 62 \% \\
\text { Domain 4: } 77 \% \\
\text { Domain 5: } 33 \% \\
\text { Domain 6: } 67 \%\end{array}$ & 8 & 15 \\
\hline $\begin{array}{l}\text { Guidelines for stress ulcer } \\
\text { prophylaxis [27] }\end{array}$ & $\begin{array}{l}\text { Strongly } \\
\quad \text { recommended }\end{array}$ & $\begin{array}{l}\text { Domain 1: } 75 \% \\
\text { Domain 2: } 46 \% \\
\text { Domain 3: } 73 \% \\
\text { Domain 4: } 73 \% \\
\text { Domain 5: } 39 \% \\
\text { Domain 6: } 63 \%\end{array}$ & 6 & 17 \\
\hline $\begin{array}{l}\text { Guidelines for the selection } \\
\text { of anti-infective agents for } \\
\text { complicated intra- } \\
\text { abdominal infections [28] }\end{array}$ & $\begin{array}{l}\text { Recommended } \\
\text { with alterations }\end{array}$ & $\begin{array}{l}\text { Domain 1: } 89 \% \\
\text { Domain 2: } 50 \% \\
\text { Domain 3: } 50 \% \\
\text { Domain 4: } 75 \% \\
\text { Domain 5: } 42 \% \\
\text { Domain 6: } 50 \%\end{array}$ & 8 & 15 \\
\hline
\end{tabular}


Appendix 1 continued

\begin{tabular}{|c|c|c|c|c|}
\hline Guideline title & $\begin{array}{l}\text { AGREE } \\
\text { recommendation }\end{array}$ & $\begin{array}{l}\text { Standardized } \\
\text { domain scores }^{\mathrm{a}}\end{array}$ & $\begin{array}{l}\text { Number of items } \\
\text { scoring } 1-2\end{array}$ & $\begin{array}{l}\text { Number of items } \\
\text { scoring 3-4 }\end{array}$ \\
\hline $\begin{array}{l}\text { Guidelines on antimicrobial } \\
\text { therapy for intra- } \\
\text { abdominal infections [29] }\end{array}$ & $\begin{array}{l}\text { Recommended } \\
\text { with alterations }\end{array}$ & $\begin{array}{l}\text { Domain 1: } 89 \% \\
\text { Domain 2: } 27 \% \\
\text { Domain 3: } 79 \% \\
\text { Domain 4: } 88 \% \\
\text { Domain 5: } 0 \% \\
\text { Domain 6: } 4 \%\end{array}$ & 9 & 14 \\
\hline $\begin{array}{l}\text { Management of critically ill } \\
\text { patients with severe acute } \\
\text { pancreatitis [30] }\end{array}$ & Not recommended & $\begin{array}{l}\text { Domain 1: } 83 \% \\
\text { Domain 2: } 29 \% \\
\text { Domain 3: } 30 \% \\
\text { Domain 4: } 67 \% \\
\text { Domain 5: } 8 \% \\
\text { Domain 6: } 8 \%\end{array}$ & 13 & 10 \\
\hline $\begin{array}{l}\text { Guidelines for the prevention } \\
\text { of intravascular catheter- } \\
\text { related infections [31] }\end{array}$ & $\begin{array}{l}\text { Recommended } \\
\text { with alterations }\end{array}$ & $\begin{array}{l}\text { Domain 1: } 72 \% \\
\text { Domain 2: } 50 \% \\
\text { Domain 3: } 32 \% \\
\text { Domain 4: } 71 \% \\
\text { Domain 5: } 42 \% \\
\text { Domain 6: } 54 \%\end{array}$ & 12 & 11 \\
\hline $\begin{array}{l}\text { Guidelines for the } \\
\text { management of } \\
\text { intravascular catheter- } \\
\text { related infections [32] }\end{array}$ & Not recommended & $\begin{array}{l}\text { Domain 1: } 67 \% \\
\text { Domain 2: } 29 \% \\
\text { Domain 3: } 29 \% \\
\text { Domain 4: } 85 \% \\
\text { Domain 5: } 25 \% \\
\text { Domain 6: } 8 \%\end{array}$ & 12 & 11 \\
\hline
\end{tabular}

a AGREE Domain 1: Scope and purpose; AGREE Domain 2: Stakeholder involvement; AGREE Domain 3: Rigor of development; AGREE Domain 4: Clarity and presentation; AGREE Domain 5: Applicability; AGREE Domain 6: Editorial independence 